\title{
Transformations between 2MASS, SDSS and BVI photometric systems for late-type giants
}

\author{
E. Yaz $^{1, \star}$, S. Bilir ${ }^{1}$, S. Karaali ${ }^{1,2}$, S. Ak ${ }^{1}$, B. Coşkunoğlu ${ }^{1}$, and A. Cabrera-Lavers ${ }^{3,4}$ \\ 1 Istanbul University, Faculty of Sciences, Department of Astronomy and Space Sciences, 34119 Istanbul, Turkey \\ 2 Beykent University, Faculty of Science and Letters, Department of Mathematics and Computer, Beykent, 34398, Istan- \\ bul, Turkey \\ 3 Instituto de Astrofísica de Canarias, E-38205 La Laguna, Tenerife, Spain \\ ${ }^{4}$ GTC Project Office, E-38205 La Laguna, Tenerife, Spain
}

The dates of receipt and acceptance should be inserted later

Key words stars: late-type, stars: general, techniques: photometric

\begin{abstract}
We present colour transformations from Two Micron All Sky Survey (2MASS) photometric system to Johnson-Cousins system and to Sloan Digital Sky Survey (SDSS) system for late-type giants and vice versa. The giant star sample was formed using surface gravity constraints $(2<\log g \leq 3)$ to Cayrel de Strobel et al.'s (2001) spectroscopic catalogue. 2MASS, SDSS and Johnson-Cousins photometric data was taken from Cutri et al. (2003), Ofek (2008) and van Leeuwen (2007), respectively. The final sample was refined applying the following steps: (1) the data were dereddened, (2) the sample stars selected are of the highest photometric quality. We give two-colour dependent transformations as a function of metallicity as well as independent of metallicity. The transformations provide absolute magnitudes and distance determinations which can be used in space density evaluations at relatively short distances where some or all of the SDSS magnitudes of late-type giants are saturated.
\end{abstract}

\section{Introduction}

The most widely used sky surveys are the Sloan Digital Sky Survey (SDSS; York et al., 2000) and the Two Micron All Sky Survey (2MASS; Skrutskie et al., 2006). SDSS is the largest photometric and spectroscopic survey in optical wavelengths, whereas, 2MASS has imaged the sky across infrared wavelengths. Another astrometrically and photometrically important survey is Hipparcos (ESA, 1997), which was reduced recently by van Leeuwen (2007).

SDSS obtains images almost simultaneously in five broadbands ( $u, g, r, i$ and $z$ ) centred at 3540, 4760, 6280, 7690 and 9250Å, respectively (Fukugita et al., 1996; Gunn et al., 1998; Hogg et al., 2001; Smith et al., 2002). The photometric pipeline (Lupton et al., 2001) detects the objects, matches the data from five filters and measures instrumental fluxes, positions and shape parameters. The magnitudes derived from fitting a point spread function (PSF) are accurate to about 2 per cent in $g, r$ and $i$, and 3-5 per cent in $u$ and $z$ for bright sources $(<20 \mathrm{mag})$ point sources. Data Release 5 (DR5) is almost 95 per cent complete for point sources to $(u, g, r, i$, $z)=(22,22.2,22.2,21.3,20.5)$. The median FWHM of the PSFs is about 1.5 arcsec (Abazajian et al., 2004). The data are saturated at about $14 \mathrm{mag}$ in $g, r$ and $i$, and about 12 mag in $u$ and $z$ (see Chonis \& Gaskell, 2008).

\footnotetext{
* Corresponding author: e-mail: esmayaz@istanbul.edu.tr
}

The near-infrared (NIR) $J H K_{s}$ photometric data were taken from the digital Two Micron All-sky Survey] (2MASS). It provides the most complete database of galactic point sources available up to date. During the development of this survey, two highly automated $1.3-\mathrm{m}$ telescopes were used: one at Mt. Hopkins, Arizona to observe the Northern sky, and the other at Cerro Tololo Observatory in Chile to complete the survey's Southern half. Observations cover 99.998 per cent (Skrutskie et al., 2006) of the sky with simultaneous detections in $J(1.25 \mu \mathrm{m}), H(1.65 \mu \mathrm{m})$ and $K_{s}(2.17$ $\mu \mathrm{m}$ ) bands up to the limiting magnitudes of $15.8,15.1$ and 14.3 , respectively. Nowadays, 2 MASS is probably the reference study for the rest of galactic surveys, due to both full coverage of the sky which it provides and its intrinsic photometric and astrometric accuracies. Recent surveys, such as UKIDSS (Lucas et al., 2008), use 2MASS as reference catalogue, therefore a transformation between optical and 2MASS magnitudes is of interest in many different topics. The photometric uncertainty of the data is less than 0.155 at $K_{s} \sim 16.5$ magnitude which is the photometric completeness of 2MASS for stars with $|b|>25^{\circ}$ (Skrutskie et al., 2006). Calibration offsets between any two points in the sky are less than $0.02 \mathrm{mag}$. The passband profiles for JohnsonCousins, SDSS and 2MASS photometric systems are given in Fig. 1.

It has been custom to derive transformations between a newly defined photometric system and those that are more traditional (such as the Johnson-Cousins $U B V R I$ system).

\footnotetext{
1 http://www.ipac.caltech.edu/2MASS/
} 

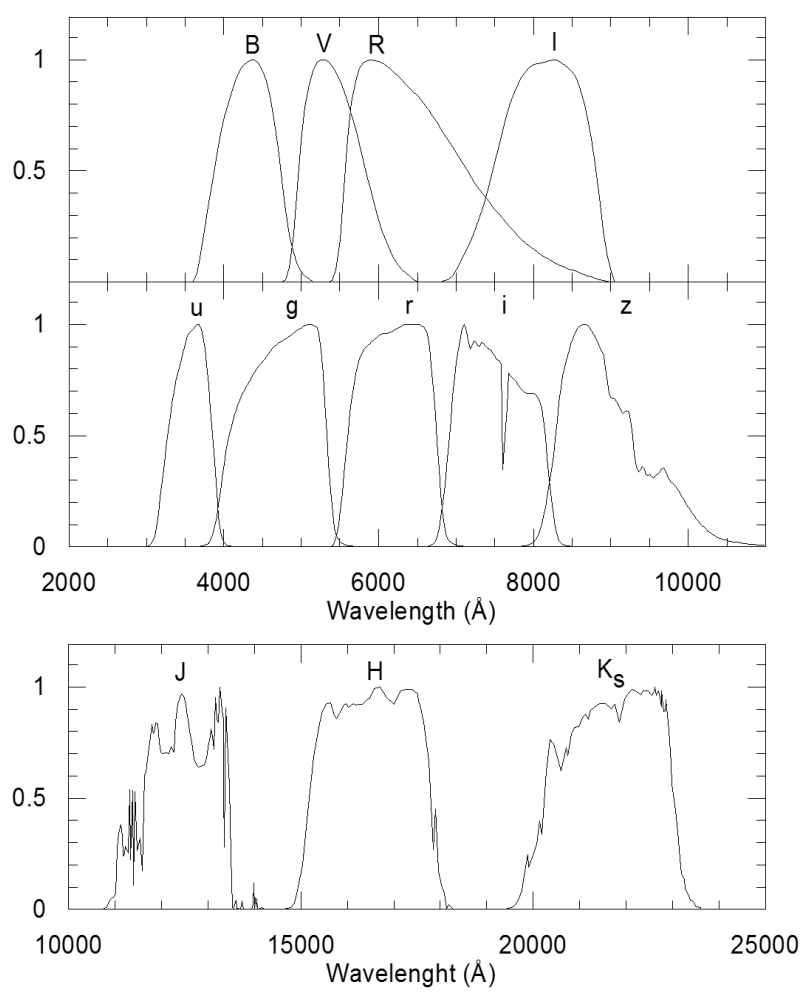

Fig. 1 Normalized passbands of the JohnsonCousins' $B V R I$ filters (upper panel), the SDSS ugriz filters (middle panel), and the 2MASS filters (lower panel).

A number of transformations between $u^{\prime} g^{\prime} r^{\prime} i^{\prime} z^{\prime}$, ugriz and $U B V R_{c} I_{c}$ exist. The $u^{\prime} g^{\prime} r^{\prime} i^{\prime} z^{\prime}$ system is referred to the similar filter system used on the 0.5-m Photometric Calibration Telescope at SDSS. It should be noted that there are differences between the $u^{\prime} g^{\prime} r^{\prime} i^{\prime} z^{\prime}$ and ugriz systems. These are discussed in Tucker et al. (2006), Davenport et al. (2007) and Smith et al. (2007). In this paper we are concerned with transformations to and from ugriz system of the $2.5-\mathrm{m}$. The first transformations derived between the SDSS $u^{\prime} g^{\prime} r^{\prime} i^{\prime} z^{\prime}$ system and the Johnson-Cousins'photometric system were based on the observations in $u^{\prime}, g^{\prime}, r^{\prime}, i^{\prime}$ and $z^{\prime}$ filters (Smith et al, 2002).

An improved set of transformations between the observations obtained in $u^{\prime} g^{\prime} r^{\prime}$ filters at the Isaac Newton Telescope (INT) at La Palma, Spain, and the Landolt (1992) $U B V$ standards is derived by Karaali et al. (2005). The INT filters were designed to reproduce the SDSS system. Karaali et (2005) presented for the first time transformation equations depending on two colors.

Rodgers et al. (2006) considered two-color or quadratic forms in their transformation equations. Jordi et al. (2006) used SDSS DR4 and $B V R I$ photometry taken from different sources and derived population (and metallicity) dependent transformation equations between SDSS and $U B V R I$ systems. A recent work is by Chonis \& Gaskell (2008) who used transformations from SDSS ugriz to $U B V R I$ not depending on luminosity class or metallicity to determine CCD zero-points. Finally, we refer to our most recent paper where transformations between SDSS (and 2MASS) and BVRI photometric systems for dwarfs are given (Bilir et al., 2008).

The first transformations between 2MASS and other photometric systems are those of Walkowicz et al. (2004) and West et al. (2005) who determined the level of magnetic activity in $\mathrm{M}$ and $\mathrm{L}$ dwarfs. The aim of Davenport et al. (2007) in deriving equations between 2MASS and other photometric systems was to estimate the absolute magnitudes of cool stars. Covey et al. (2008) considered the ugrizJH $K_{s}$ stellar locus and showed how it can be used to identify objects with unusual colors. A recent study by Straizys \& Lazauskaite (2009) provided calibrations which can be used to obtain the color indices of 2MASS stars with known spectral types and luminosity classes. Straizys \& Lazauskaite (2009) used the loci of giant stars with spectral types later than G5 to obtain their calibrations.

To extend the results of Bilir et al. (2008) to the giants domain is of great use, as it allows to derive absolute magnitudes and to produce distance determinations at relatively short distances where SDSS sources will be saturated. For example, red clump giants are now considered as well-known standard candles, as they show a very narrow luminosity function that constitute a compact and well-defined clump in a Hertzsprung-Russell diagram, particularly in the NIR. The absolute magnitude $\left(M_{K}\right)$ and intrinsic colors, $(J-$ $\left.K_{s}\right)_{0}$, of the red clump giants are well established with very small metallicity dependences (see Cabrera-Lavers et al., 2008, and references therein), hence distances to these sources can be derived with confidence by means of a very small number of assumptions (López-Corredoira et al., 2002). This well known set of NIR properties of the red clump population have not been sufficiently studied in the optical, where a lot of SDSS data is waiting to be exploited. In order to do this, a proper set of optical/NIR photometric transformations are needed and these are presented here.

In Section 2 we present the sources of our star sample and the criteria applied to the chosen stars. The transformation equations are given in Section 3. Finally, in Section 4, we discuss our results.

\section{Data}

The first and main source of our data was Cayrel de Strobel et al.'s (2001) spectroscopic catalogue. This catalogue conal.ins a large number of stars with different population types and metallicities. We chose 661 stars with $2<\log g \leq 3$ and obtained the original sample of late-type giants. The second source for our work is the 2MASS All-Sky Catalogue of Point Sources (Cutri et al., 2003). We matched the Cayrel de Strobel et al.'s (2001) spectroscopic catalogue with the Cutri et al.'s (2003) 2MASS photometric catalogue and obtained the NIR magnitudes for 661 stars. The NIR magnitudes of 661 stars are not as precise as their optical magnitudes. To select more sensitive NIR magnitudes of sample stars, we used the magnitude flags, labeled "AAA", 

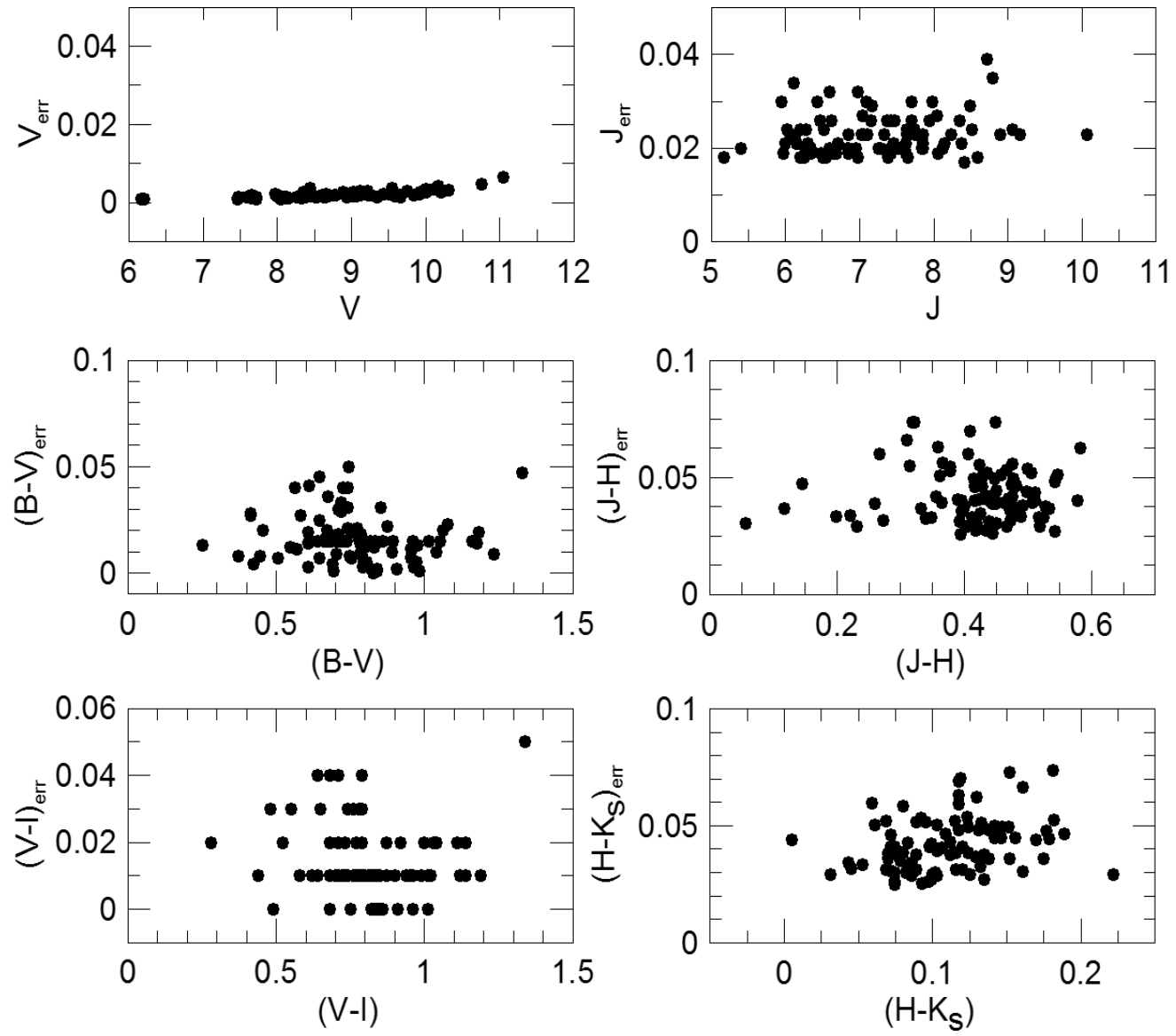

Fig. 2 The error distributions for Johnson-Cousins' $B V I$ and 2MASS $J H K_{s}$ (the errors for SDSS gri magnitudes are discussed in the text).

which means the signal noise ratio is $S N R \geq 10$, i.e. they have the highest quality measurements.

After applying this selection criterion based on the quality of the data, the total number of stars in 2MASS photometric system was reduced to 91 . The photometric and spectroscopic data of 91 stars is given in Table 1.

The BVI magnitudes were taken from the newly reduced Hipparcos' (van Leeuwen, 2007) catalogue. The same catalogue also offers trigonometric parallaxes which provides accurate distance determination. Finally, the $g, r$ and $i$ magnitudes were taken from the Ofek's (2008) catalogue. Ofek (2008) calculated the synthetic griz magnitudes of bright Tycho stars, where SDSS magnitudes are actually saturated. Out of 91 stars only 82 had $g, r$ and $i$ magnitudes, due to the shallowness of 2MASS and deepness of the SDSS photometries. Thus, transformation equations between $B V I$ and 2MASS is carried out for a sample of 91 stars, whereas the ones between SDSS and 2MASS is limited with 82 stars.

The errors for the colors and magnitudes for $B V I$ and 2MASS photometries are given in Fig. 2. For SDSS magnitudes, Ofek (2008) states that the typical errors for a single star are $0.12,0.12,0.10$ and 0.08 mag for $g, r, i$ and $z$ bands, respectively, but they reduce to about $0.04,0.03,0.02$ and $0.02 \mathrm{mag}$ for a sample of about 10 stars.

The $E(B-V)$ color excesses of stars have been evaluated in two steps. First, we used the maps of Schlegel et al. (1998) and evaluated a $E_{\infty}(B-V)$ color excess for each star. We then reduced them using the following procedure (Bahcall \& Soneira, 1980):

$$
A_{d}(b)=A_{\infty}(b)\left[1-\exp \left(\frac{-|d \sin (b)|}{H}\right)\right] .
$$

Here, $b$ and $d$ are the galactic latitude and distance of the star, respectively. $H$ is the scaleheight for the interstellar dust which is adopted as $125 \mathrm{pc}$ (Marshall et al., 2006) and $A_{\infty}(b)$ and $A_{d}(b)$ are the total absorptions for the model and for the distance to the star, respectively. $A_{\infty}(b)$ can be evaluated by means of the following equation:

$$
A_{\infty}(b)=3.1 E_{\infty}(B-V) .
$$

$E_{\infty}(B-V)$ is the color excess for the model taken from the NASA Extragalactic Database. Then, $E_{d}(B-V)$, i.e. the color excess for the corresponding star at the distance $d$, can be evaluated by Eq. (3) adopted for distance $d$,

$$
E_{d}(B-V)=A_{d}(b) / 3.1 .
$$

\footnotetext{
${ }^{2}$ http://nedwww.ipac.caltech.edu/forms/calculator.html
} 
Table 1 Johnson-Cousins, SDSS and 2MASS magnitudes, coordinates and parallax of the sample stars (91 total stars). The columns give: (1) ID, (2) Hipparcos number, (3)-(5) surface gravity, metal abundance and their references (6) parallax, (7) relative parallax error, (8) distance (9) $E_{d}(B-V)$ reduced color excess, (10) $V$ apparent magnitude, (11) and (12) $B-V$ and $V-I$ color indices, (13) $g$ apparent magnitude, (14) and (15) $g-r, r-i$ color indices, (16) $J$ apparent magnitude, (17) and (18) $J-H$ and $H-K_{s}$ color indices.

\begin{tabular}{|c|c|c|c|c|c|c|c|c|c|c|c|c|c|c|c|c|c|}
\hline ID & Hip 1 & $\log g$ & 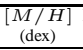 & & $\begin{array}{c}\pi \\
\text { (mas) }\end{array}$ & $\sigma_{\pi / \pi}$ & $\begin{array}{c}d \\
(\mathrm{pc})\end{array}$ & $E_{(\mathrm{mag})}^{E_{d}^{(B-V)}}$ & $\begin{array}{c}V \\
(\mathrm{mag}) \\
\end{array}$ & $\begin{array}{c}B \begin{array}{c}B-V \\
(\mathrm{mag})\end{array} \\
\end{array}$ & $\begin{array}{l}V-I \\
\text { (mag) }\end{array}$ & $\begin{array}{c} \\
(\mathrm{mag})\end{array}$ & $\begin{array}{l}g-r \\
(\mathrm{mag}) \\
\end{array}$ & $\begin{array}{c}r-i \\
(\mathrm{mag})\end{array}$ & $\begin{array}{c}J \\
(\mathrm{mag}) \\
\end{array}$ & $\begin{array}{l}J-H \\
(\mathrm{mag}) \\
\end{array}$ & $\begin{array}{c}H-K_{s} \\
(\mathrm{mag})\end{array}$ \\
\hline & $\begin{array}{l}434 \\
447\end{array}$ & $\begin{array}{l}2.80 \\
250\end{array}$ & $\begin{array}{l}-1.39 \\
-045\end{array}$ & & & 0.43 & 334 & $\begin{array}{l}0.016 \\
074\end{array}$ & $9.040 \pm 0.002$ & & & 8790 & $\begin{array}{l}0.480 \\
0730\end{array}$ & & & & \\
\hline & $\begin{array}{l}447 \\
484\end{array}$ & $\begin{array}{l}2.50 \\
260\end{array}$ & $\begin{array}{l}-0.45 \\
-1.19\end{array}$ & & $\begin{array}{l}2.99 \pm 1.16 \\
0.37+1.33\end{array}$ & $\begin{array}{l}0.39 \\
3.59\end{array}$ & $\begin{array}{l}334 \\
540 *\end{array}$ & 0.074 & $8.260 \pm 0.002$ & $1.052 \pm 0.015$ & & 8.790 & 0.730 & 0.270 & $6.540 \pm 0$ & & \\
\hline & 999 & $\begin{array}{l}2.00 \\
3.00\end{array}$ & $\begin{array}{l}-1.19 \\
-0.40\end{array}$ & & $\begin{array}{l}0.37 \pm 1.33 \\
24.38 \pm 0.95\end{array}$ & & $\begin{array}{l}544^{*} \\
41\end{array}$ & $\begin{array}{l}0.021 \\
0.011\end{array}$ & $\begin{array}{l}660 \pm 0.002 \\
440 \pm 0.004\end{array}$ & & & & & $\begin{array}{l}0.180 \\
0.250\end{array}$ & & & \\
\hline & 1298 & 2.60 & -1.17 & & \pm 1.67 & & $520^{*}$ & 0.020 & \pm 0.0 & & & 9.850 & 0.480 & 0.150 & & & .051 \\
\hline & 2023 & 2.50 & -0.11 & 5 & $7.29 \pm 0$ & 0.10 & 137 & 0.021 & $8.000 \pm 0$. & $0.253 \pm 0.0$ & $0.28 \pm 0.02$ & 8.160 & 0.120 & 0.000 & $7.415 \pm 0$ & & \\
\hline & $\begin{array}{l}2413 \\
2463\end{array}$ & $\begin{array}{l}2.50 \\
2.25\end{array}$ & $\begin{array}{l}-2.00 \\
-2.10\end{array}$ & $\begin{array}{l}6 \\
7 \\
\end{array}$ & & . & $\begin{array}{l}317 \\
313^{*}\end{array}$ & $\begin{array}{l}0.086 \\
0.019\end{array}$ & $\begin{array}{l}20 \pm 0.001 \\
180+0.002\end{array}$ & $\begin{array}{l}0.747 \pm 0.008 \\
0.713 \pm 0.015\end{array}$ & & 8.800 & 0.660 & 0.260 & $\begin{array}{l}6.026 \pm 0 \\
6.853 \pm 0\end{array}$ & & \\
\hline & 2727 & 2.60 & -1.04 & 3 & $3.47 \pm 1.24$ & 0.36 & 288 & 0.018 & $750 \pm 0.003$ & $0.735 \pm 0.015$ & $0.78 \pm 0.01$ & $\begin{array}{l}0.080 \\
10.080\end{array}$ & 0.480 & 0.150 & $76 \pm 0.021$ & & $.106 \pm 0.041$ \\
\hline 10 & 3554 & 3.00 & -2.30 & 7 & $5.01 \pm 1.32$ & 0.2 & 200 & & $20 \pm 0.003$ & $0.745 \pm 0.0$ & & & 0. & & & & \\
\hline & $\begin{array}{l}4930 \\
4960\end{array}$ & $\begin{array}{l}2.80 \\
2.53\end{array}$ & & $\begin{array}{l}8 \\
9\end{array}$ & & & $\begin{array}{l}386 \\
314\end{array}$ & & & & & & & & & & \\
\hline & $\begin{array}{l}4960 \\
5104\end{array}$ & $\begin{array}{l}2.33 \\
2.30\end{array}$ & $\begin{array}{l}-0.99 \\
-0.93\end{array}$ & $\begin{array}{c}9 \\
10\end{array}$ & $\begin{array}{l} \pm 0 . \\
\pm 0 .\end{array}$ & 15 & $\begin{array}{l}314 \\
313\end{array}$ & $\begin{array}{l}0.027 \\
0.023\end{array}$ & $\begin{array}{l}0 \pm 0.002 \\
0+0.002\end{array}$ & $\begin{array}{l}3 \pm \pm 0 \\
35 \pm 0\end{array}$ & $\begin{array}{l}9 \pm 0.02 \\
2 \pm 0.01\end{array}$ & $\begin{array}{l}9.050 \\
8.090\end{array}$ & $\begin{array}{l}0.640 \\
0.640\end{array}$ & $\begin{array}{l}0.210 \\
0.210\end{array}$ & $\begin{array}{l}10 \\
\pm 0\end{array}$ & & $\begin{array}{l}031 \\
031\end{array}$ \\
\hline 5 & 5445 & 2.50 & -1.56 & 6 & \pm 0.84 & 0.12 & 140 & 221 & \pm 0.002 & & & 8.090 & 0.630 & & & & \\
\hline & 5455 & & & 9 & & & 138 & & & & & & & & & & \\
\hline & 68994 & & & 9 & & & 39 & & & & & & & & & & \\
\hline & $\begin{array}{l}12028 \\
12353\end{array}$ & 年.10 & $=0$ & 11 & & & 6. & & & & & 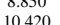 & 0. & & & & \\
\hline & 14747 & & & 7 & & & & & & & & & & & & & \\
\hline & & & & 13 & & & & & & & & & & & & & \\
\hline & & & & 14 & & & 25 & & 2 & & & & & & & 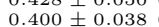 & \\
\hline & & & & & 2 & & 392 & & 22 & & & & & & & & \\
\hline & 21473 & & & 10 & \pm 0 & & $449^{*}$ & & 22 & & 1 & & 0.720 & & & & \\
\hline & & & & 15 & & & 2.3 & & & & & & & & & & \\
\hline & 68487 & $\begin{array}{l}3.00 \\
3.00\end{array}$ & $\begin{array}{l}-1 . \\
-1.6 \\
0\end{array}$ & 7 & $\begin{array}{l} \pm 0 . \\
\pm 1 .\end{array}$ & & $728 *$ & & 22 & & & 9.810 & & & & & \\
\hline & & & & & & & & & $\frac{3}{2}$ & & & & & & & & \\
\hline & 20992 & 2.20 & & & a & & & & & & & & & & & & \\
\hline & 30668 & 3.00 & & & & & & & & & & & & & & & \\
\hline & & 2.80 & & & 0 & & & & & & & & & & & & \\
\hline & 7335. & 3.00 & & & 1 & & & & & & & & & & & & \\
\hline & & & & & 0 & & & & & & & & 240 & 010 & & & \\
\hline & & & & & & & & & & & & & & & & & \\
\hline & 然16 & . & & & & & & & & & & & & & & & \\
\hline & 45845 & 2.3 & & 10 & 3 & & & & & & & & & & & & \\
\hline & & & & & & & & & & & & & & & & & \\
\hline & 4879 & 220 & $\begin{array}{l}-1.40 \\
-245 \\
-2.04\end{array}$ & $\begin{array}{c}23 \\
6\end{array}$ & & & 61 & & & & & 9.570 & 0.480 & 0.150 & & & \\
\hline & & $\begin{array}{l}2.20 \\
2.10\end{array}$ & & & 0 & & & & 2 & & & & & & & & \\
\hline & & 2.58 & & 24 & -1 & & & & & & & & & & & & \\
\hline & & & & 12 & & & & & & & & & & & & & \\
\hline & & $2.80 \mathrm{Y}>\mathrm{C}$ & & & & & & & & & & & & & & & \\
\hline & & 2.10 & & & & & & & & & & & & & & & \\
\hline & & 2.60 & & & & & & & & & & & & & & & \\
\hline & & & & & & & & & & & & & & & & & \\
\hline & & $\begin{array}{l}2.20 \\
2.20\end{array}$ & $\begin{array}{l}-1.12 \\
-0.59\end{array}$ & 11 & \pm 0 & & $\begin{array}{l}398 \\
290\end{array}$ & & & & & & - & 0.260 & $\begin{array}{l}6 . \\
7 .\end{array}$ & & \\
\hline & & 2.60 & -1.04 & & \pm 1 & & $520^{*}$ & & 10 & & & & & 0.26 & & & \\
\hline & & 2.50 & -2.25 & & \pm 1.10 & & $522^{*}$ & 0.021 & $9.590 \pm 0.002$ & & & 9.960 & 0.610 & 0.210 & $8.126 \pm 0.020$ & $0.409 \pm 0.070$ & $0.119 \pm 0.070$ \\
\hline
\end{tabular}




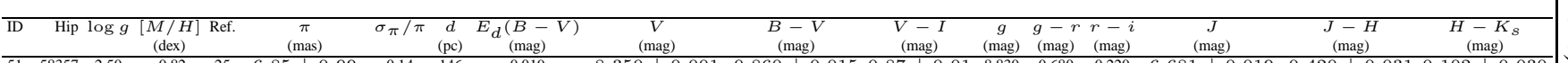

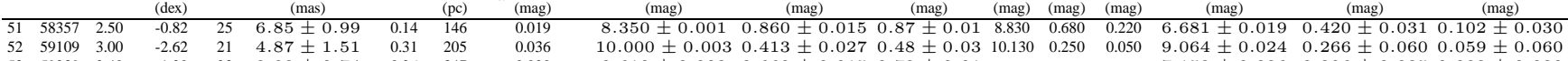

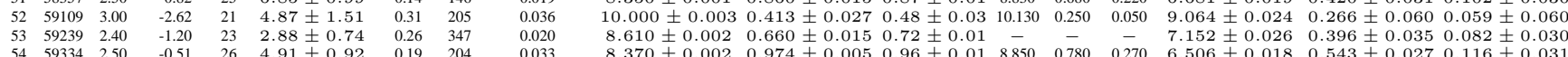

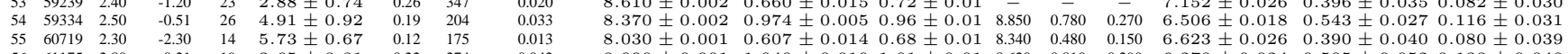

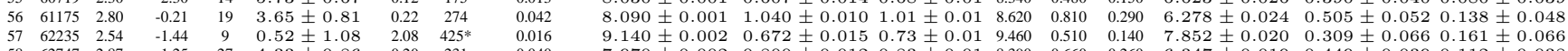

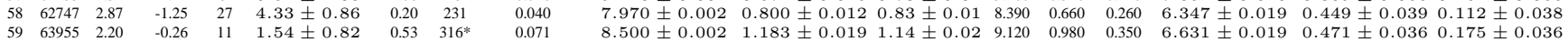

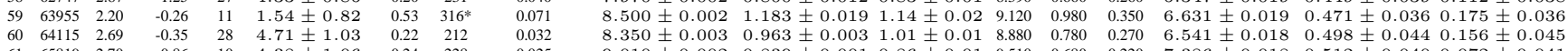

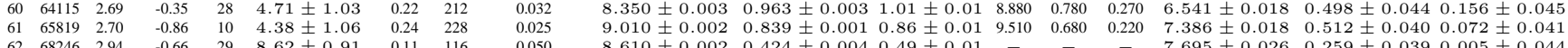

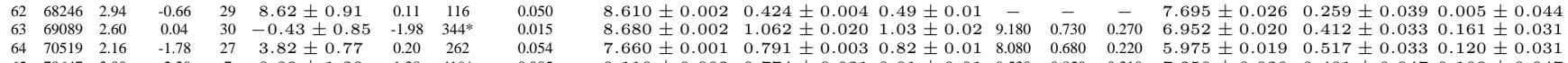

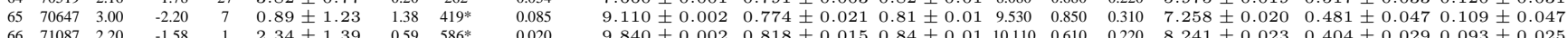

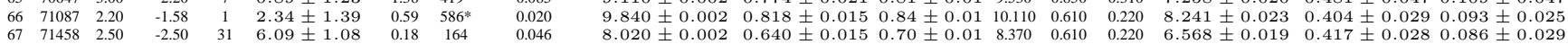

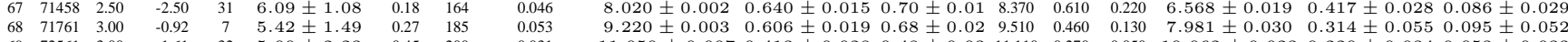

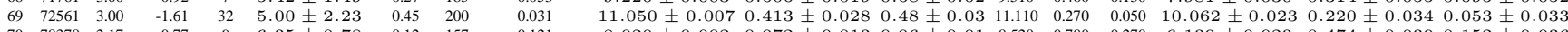

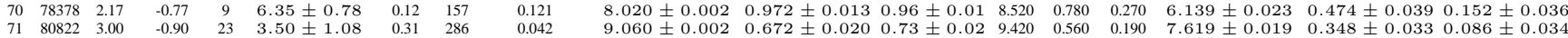

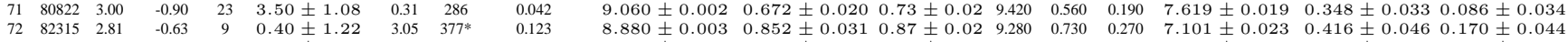

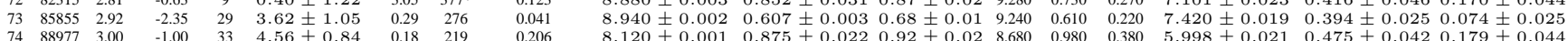

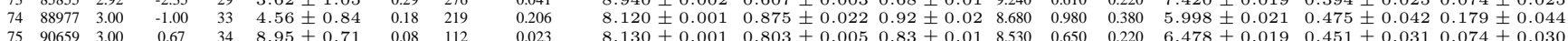
$7.590 \pm 0.0010 .5480 .0120 .62 \pm 0.017850 \quad 0500-0.220 \quad 6.478 \pm 0.019-0.451 \pm 0.0310 .074 \pm 0.030$

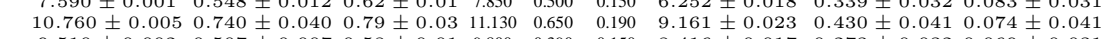

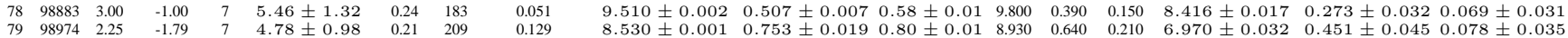

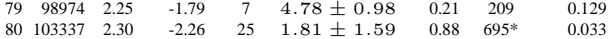

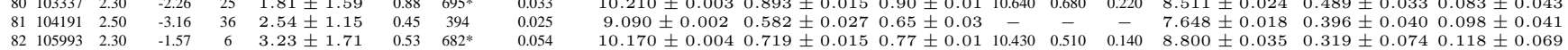

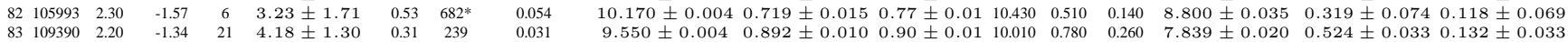

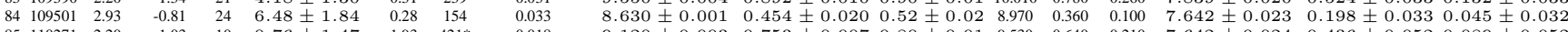

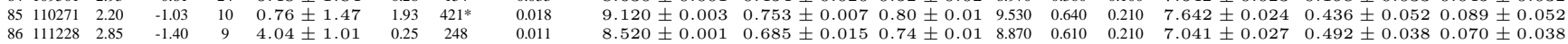

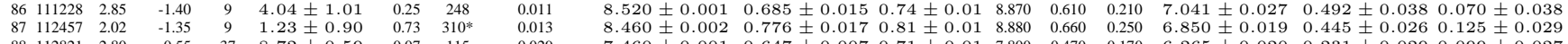
\begin{tabular}{llllllllllllllllll}
88 & 112821 & 2.80 & -0.55 & 37 & $8.72 \pm 0.59$ & 0.07 & 115 & 0.029 & $7.460 \pm 0.001$ & $0.647 \pm 0.007$ & $0.71 \pm 0.01$ & 7.800 & 0.470 & 0.170 & $6.265 \pm 0.020$ & $0.231 \pm 0.029$ & $0.099 \pm 0.027$ \\
\hline
\end{tabular} \begin{tabular}{llllllllllllllllll}
89 & 114502 & 2.40 & -1.86 & 1 & $3.21 \pm 1.09$ & 0.34 & 312 & 0.032 & $8.940 \pm 0.002$ & $0.718 \pm 0.029$ & $0.77 \pm 0.02$ & 9.270 & 0.630 & 0.210 & $7.397 \pm 0.019$ & $0.406 \pm 0.060$ & $0.118 \pm 0.059$ \\
90 & 116285 & 2.49 & -1.14 & 9 & $0.76 \pm 1.28$ & 1.68 & $433^{*}$ & 0.009 & $9.180 \pm 0.002$ & $0.689 \pm 0.004$ & $0.75 \pm 0.01$ & 9.500 & 0.610 & 0.220 & $7.736 \pm 0.024$ & $0.366 \pm 0.056$ & 0.123 \\
\hline
\end{tabular}

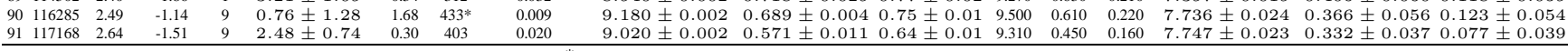
$\left(^{*}\right)$ The derived distance using the method explained in this paper

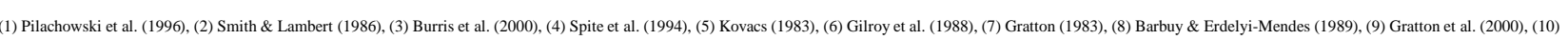

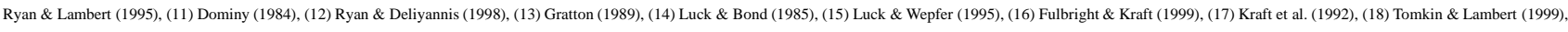

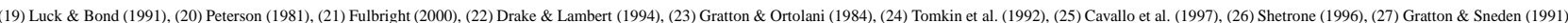

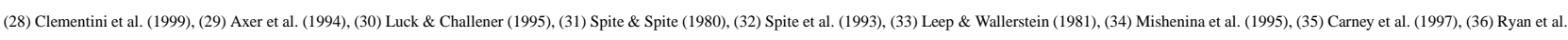
[1991), (37) [rishnaswamv \& Sneden [1985). 
We have omitted the indices $\infty$ and $d$ from the color excess $E(B-V)$ in the equations. However, we use the terms model for the color excess of Schlegel et al. (1998) and "reduced" the color excess corresponding to distance $d$. The total absorption $A_{d}$ used in this section and the classical total absorption $A_{V}$ have the same meaning.

The distances of 65 stars whose relative parallax errors are small were evaluated by using their original parallaxes. However, for one star with negative trigonometric parallax and for 25 stars with relative parallax errors $\sigma_{\pi} / \pi>0.5$ the following formula was used for this purpose:

$$
V-M_{V}-A_{d}(b)=5 \log d-5 .
$$

The absolute magnitudes for this sub-sample of stars were adopted as $M_{V}=1$. The distances derived using this method are denoted with a star superscript in Table 1 . As the total absorptions for the model and distance to the star are different, the distance to a star in this category (a total of 26 stars), as well as its total absorption, $A_{d}(b)$, and color excess, $E_{d}(B-V)$, could be evaluated by iterating Eqs. (1)(4).

We de-reddened the colors and magnitudes by using the $E_{d}(B-V)$ color index of the stars evaluated using the procedures explained above and the equations of Fan (1999); Fiorucci \& Munari (2003); Yadav \& Sagar (2004) for $V-I$ color, and for 2MASS and SDSS photometries, respectively. The related equations are given in the following:

$$
\begin{array}{r}
V_{0}=V-3.1 E_{d}(B-V), \\
(B-V)_{0}=(B-V)-E_{d}(B-V), \\
(V-I)_{0}=(V-I)-1.250 E_{d}(B-V), \\
J_{0}=J-0.887 E_{d}(B-V), \\
(J-H)_{0}=(J-H)-0.322 E_{d}(B-V), \\
\left(H-K_{s}\right)_{0}=\left(H-K_{s}\right)-0.183 E_{d}(B-V), \\
g_{0}=g-1.199 E_{d}(B-V), \\
(g-r)_{0}=(g-r)-0.341 E_{d}(B-V), \\
(r-i)_{0}=(r-i)-0.219 E_{d}(B-V) .
\end{array}
$$

The two color diagrams of the star sample for three photometric systems, i.e. $B V I$, gri and $J H K_{s}$, compared to the calibrations of Pickles (1998), Covey et al. (2008) and Straizys \& Lazauskaite (2009) are given in Fig. 3, respectively. The valid color index intervals for the transformations for giants are as follows: $0.25<(B-V)_{0}<1.35$, $0.25<(V-I)_{0}<1.35,0.10<(g-r)_{0}<0.95$, $0<(r-i)_{0}<0.35,0.05<(J-H)_{0}<0.60,0<$ $\left(H-K_{s}\right)_{0}<0.45$. The metallicity distribution of the sample covers all three populations, i.e. thin and thick disks, and halo (Fig. 4). The modes for the distributions of the whole sample (91 stars) and for the sample of stars ( 82 stars) which do not supply the relative parallax error condition $\left(\sigma_{\pi} / \pi<0.5\right)$ are -1.23 and -1.21 dex, respectively. The corresponding medians of these distributions are close to the modes (-1.18 and -1.12 dex) indicating two Gaussian distributions.
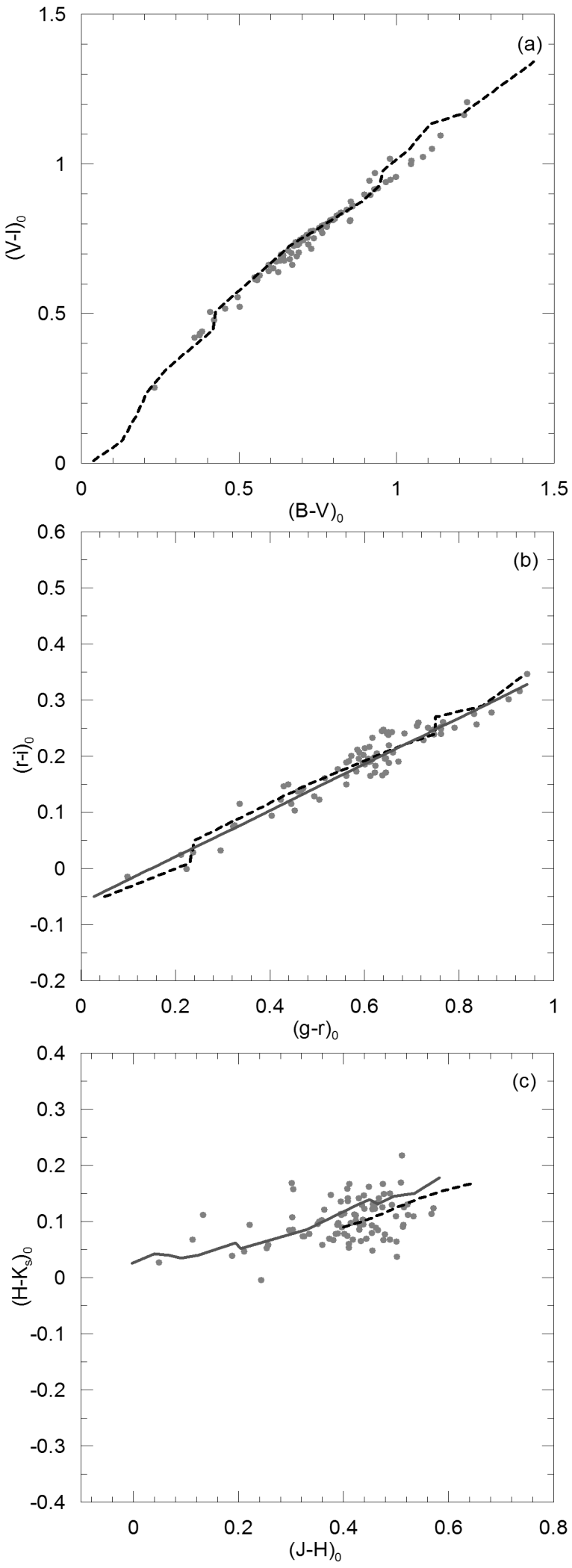

Fig. 3 Two-color diagrams of the sample stars. In all panels, the grey circles correspond to the positions of our sample stars. The dashed lines in panels (a), (b) and (c) are the calibrations of Pickles (1998), Covey et al. (2008), and Straizys \& Lazauskaite (2009), respectively. (The continuous lines in panels (b) and (c) correspond to the two-color calibrations evaluated by the converted colors in Table 4 . See section 4). 


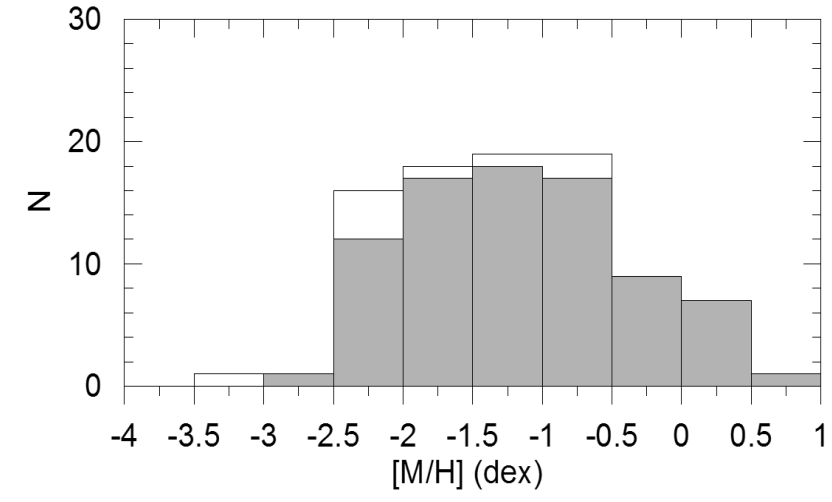

Fig. 4 Metalicity distributions for two samples: (1) for 91 late-type giant stars used in the transformations between $B V I$ and $J H K_{s}$ magnitudes (white area) and (2) for 82 giant stars used in the transformations between $\mathrm{gri}$ and $J H K_{s}$ magnitudes (grey area).

\section{Results}

\subsection{Transformations between 2MASS and Johnson-Cousins photometry}

We used the following general equations and derived 12 sets of transformations between 2MASS and Johnson-Cousins $B V I$. Eqs. (6)-(11) transform 2MASS colors and magnitudes into $B V I$ magnitudes, whereas Eqs. (12)-(17) are their inverse transformations. The transformations are either metallicity dependent (Eqs. (6)-(8) and (12)-(14)) or independent of metallicity (Eqs. (9)-(11) and (15)-(17)). In this work, we followed a procedure different than the one in Bilir et al. (2008), used for the dwarfs. Bilir et al. (2008) separated the sample of stars into metal-rich, intermediate metallicity and metal-poor sub-samples and obtained transformations for each sub-sample, whereas we adopted the metallicity as an additional term in Eqs. (6)-(8) and (12)(14). This approach can be explained by the fact that stars change their positions in two color diagrams by shifting an amount proportional to their metallicities. The general equations are

$$
\begin{aligned}
& (B-J)_{0}=a_{1}(J-H)_{0}+b_{1}\left(H-K_{s}\right)_{0}+c_{1}[M / H]+d_{1},(6) \\
& (V-J)_{0}=a_{2}(J-H)_{0}+b_{2}\left(H-K_{s}\right)_{0}+c_{2}[M / H]+d_{2},(7) \\
& (I-J)_{0}=a_{3}(J-H)_{0}+b_{3}\left(H-K_{s}\right)_{0}+c_{3}[M / H]+d_{3},(8) \\
& (B-J)_{0}=a_{4}(J-H)_{0}+b_{4}\left(H-K_{s}\right)_{0}+d_{4}, \\
& (V-J)_{0}=a_{5}(J-H)_{0}+b_{5}\left(H-K_{s}\right)_{0}+d_{5}, \\
& (I-J)_{0}=a_{6}(J-H)_{0}+b_{6}\left(H-K_{s}\right)_{0}+d_{6}, \\
& (V-J)_{0}=e_{1}(B-V)_{0}+f_{1}(V-I)_{0}+g_{1}[M / H]+h_{1},(12) \\
& (V-H)_{0}=e_{2}(B-V)_{0}+f_{2}(V-I)_{0}+g_{2}[M / H]+h_{2},(13) \\
& \left(V-K_{s}\right)_{0}=e_{3}(B-V)_{0}+f_{3}(V-I)_{0}+g_{3}[M / H]+h_{3},(14) \\
& (V-J)_{0}=e_{4}(B-V)_{0}+f_{4}(V-I)_{0}+h_{4}, \\
& (V-H)_{0}=e_{5}(B-V)_{0}+f_{5}(V-I)_{0}+h_{5}, \\
& \left(V-K_{s}\right)_{0}=e_{6}(B-V)_{0}+f_{6}(V-I)_{0}+h_{6} .
\end{aligned}
$$

The numerical values of the coefficients in Eqs. (6)-(17) are given in Table 2.
Table 1 Coefficients for the transformation Eqs. (6)-(17) in column matrix. The subscript $i=1,2$ and 3 or $i=4,5$ and 6 correspond to the same number that denotes the equations. $R$ and $s$ are the correlation coefficient and standard deviation for each category, respectively.

\begin{tabular}{cccc}
\hline \multicolumn{4}{c}{ Equations dependent on metallicity, $i=1,2,3$} \\
Coefficient & $(B-J)_{0}$ & $(V-J)_{0}$ & $(I-J)_{0}$ \\
\hline$a_{i}$ & $3.732 \pm 0.163$ & $2.328 \pm 0.102$ & $1.097 \pm 0.089$ \\
$b_{i}$ & $2.125 \pm 0.312$ & $1.128 \pm 0.195$ & $0.306 \pm 0.170$ \\
$c_{i}$ & $0.114 \pm 0.019$ & $0.033 \pm 0.012$ & $-0.031 \pm 0.011$ \\
$d_{i}$ & $0.592 \pm 0.071$ & $0.435 \pm 0.044$ & $0.185 \pm 0.038$ \\
$R$ & 0.950 & 0.945 & 0.832 \\
$s$ & 0.142 & 0.089 & 0.077 \\
\hline \multicolumn{5}{c}{ Equations independent from metallicity, $i=4,5,6$} \\
Coefficient & $(B-J)_{0}$ & $(V-J)_{0}$ & $(I-J)_{0}$ \\
\hline$a_{i}$ & $3.671 \pm 0.191$ & $2.310 \pm 0.105$ & $1.114 \pm 0.092$ \\
$b_{i}$ & $2.518 \pm 0.359$ & $1.242 \pm 0.198$ & $0.198 \pm 0.173$ \\
$d_{i}$ & $0.436 \pm 0.077$ & $0.390 \pm 0.042$ & $0.228 \pm 0.037$ \\
$R$ & 0.929 & 0.940 & 0.812 \\
$s$ & 0.167 & 0.092 & 0.080 \\
\hline \multicolumn{5}{c}{ Equations dependent on metallicity, $i=1,2,3$} \\
Coefficient & $(V-J)_{0}$ & $(V-H)_{0}$ & $\left(V-K_{s}\right)_{0}$ \\
\hline$e_{i}$ & $1.080 \pm 0.433$ & $1.454 \pm 0.590$ & $1.791 \pm 0.592$ \\
$f_{i}$ & $0.379 \pm 0.495$ & $0.504 \pm 0.674$ & $0.294 \pm 0.676$ \\
$g_{i}$ & $-0.082 \pm 0.015$ & $-0.127 \pm 0.020$ & $-0.129 \pm 0.020$ \\
$h_{i}$ & $0.279 \pm 0.078$ & $0.261 \pm 0.106$ & $0.276 \pm 0.106$ \\
$R$ & 0.933 & 0.931 & 0.940 \\
$s$ & 0.097 & 0.133 & 0.133 \\
\hline Equations independent from metallicity, $i=4,5,6$ \\
Coefficient & $(V-J)_{0}$ & $(V-H)_{0}$ & $\left(V-K_{s}\right)_{0}$ \\
\hline$e_{i}$ & $0.397 \pm 0.484$ & $0.402 \pm 0.684$ & $0.716 \pm 0.690$ \\
$f_{i}$ & $1.018 \pm 0.560$ & $1.488 \pm 0.792$ & $1.299 \pm 0.799$ \\
$h_{i}$ & $0.396 \pm 0.087$ & $0.441 \pm 0.123$ & $0.460 \pm 0.124$ \\
$R$ & 0.907 & 0.896 & 0.908 \\
$s$ & 0.113 & 0.160 & 0.162 \\
\hline \multicolumn{5}{c}{$(V)$}
\end{tabular}

\subsection{Transformations between 2MASS and SDSS}

The transformations between 2MASS and SDSS, given in the following, have similar general equations:

$$
\begin{aligned}
& (g-J)_{0}=k_{1}(J-H)_{0}+l_{1}\left(H-K_{s}\right)_{0}+m_{1}[M / H]+n_{1} .(18) \\
& (r-J)_{0}=k_{2}(J-H)_{0}+l_{2}\left(H-K_{s}\right)_{0}+m_{2}[M / H]+n_{2} .(19) \\
& (i-J)_{0}=k_{3}(J-H)_{0}+l_{3}\left(H-K_{s}\right)_{0}+m_{3}[M / H]+n_{3} \cdot(20) \\
& (g-J)_{0}=k_{4}(J-H)_{0}+l_{4}\left(H-K_{s}\right)_{0}+n_{4} \cdot \\
& (r-J)_{0}=k_{5}(J-H)_{0}+l_{5}\left(H-K_{s}\right)_{0}+n_{5} \cdot \\
& (i-J)_{0}=k_{6}(J-H)_{0}+l_{6}\left(H-K_{s}\right)_{0}+n_{6} \cdot \\
& (g-J)_{0}=o_{1}(g-r)_{0}+p_{1}(r-i)_{0}+r_{1}[M / H]+s_{1} \cdot(24) \\
& (g-H)_{0}=o_{2}(g-r)_{0}+p_{2}(r-i)_{0}+r_{2}[M / H]+s_{2} \cdot(25) \\
& \left(g-K_{s}\right)_{0}=o_{3}(g-r)_{0}+p_{3}(r-i)_{0}+r_{3}[M / H]+s_{3} \cdot(26) \\
& (g-J)_{0}=o_{4}(g-r)_{0}+p_{4}(r-i)_{0}+s_{4} . \\
& (g-H)_{0}=o_{5}(g-r)_{0}+p_{5}(r-i)_{0}+s_{5} . \\
& \left(g-K_{s}\right)_{0}=o_{6}(g-r)_{0}+p_{6}(r-i)_{0}+s_{6} .
\end{aligned}
$$

One can see that, the equations which convert 2MASS colors and magnitudes into gri magnitudes and their inverse transformations are either metallicity dependent or independent of metallicity. The numerical values of the coefficients in Eqs. (18)-(29) are given in Table 3. 
Table 2 Coefficients for the transformation Eqs. (18)(29) in column matrix. The subscript $i=1,2$ and 3 or $i$ $=4,5$ and 6 correspond to the same number that denotes the equations. $R$ and $s$ are the correlation coefficient and standard deviation for each category, respectively.

\begin{tabular}{|c|c|c|c|}
\hline \multicolumn{4}{|c|}{ Equations dependent on metallicity, $i=1,2,3$} \\
\hline Coefficient & $(g-J)_{0}$ & $(r-J)_{0}$ & $(i-J)_{0}$ \\
\hline$k_{i}$ & $2.992 \pm 0.127$ & $1.743 \pm 0.087$ & $1.233 \pm 0.083$ \\
\hline$l_{i}$ & $2.478 \pm 0.357$ & $1.250 \pm 0.245$ & $0.727 \pm 0.232$ \\
\hline$m_{i}$ & $0.089 \pm 0.016$ & $0.054 \pm 0.011$ & $0.042 \pm 0.010$ \\
\hline$n_{i}$ & $0.461 \pm 0.057$ & $0.462 \pm 0.039$ & $0.524 \pm 0.037$ \\
\hline$R$ & 0.960 & 0.943 & 0.901 \\
\hline$s$ & 0.106 & 0.073 & 0.069 \\
\hline \multicolumn{4}{|c|}{ Equations independent from metallicity, $i=4,5,6$} \\
\hline & $(g-J)_{0}$ & $(r-J)_{0}$ & $(i-J)_{0}$ \\
\hline$k_{i}$ & $2.923 \pm 0.148$ & $1.701 \pm 0.099$ & $1.200 \pm 0.090$ \\
\hline$l_{i}$ & $3.031 \pm 0.400$ & $1.585 \pm 0.266$ & $0.991 \pm 0.243$ \\
\hline$n_{i}$ & $0.329 \pm 0.061$ & $0.383 \pm 0.041$ & $0.461 \pm 0.037$ \\
\hline$R$ & 0.943 & 0.925 & 0.879 \\
\hline$s$ & 0.124 & 0.083 & 0.075 \\
\hline \multicolumn{4}{|c|}{ Equations dependent on metallicity, $i=1,2,3$} \\
\hline & $(g-J)_{0}$ & $(g-H)_{0}$ & $\left(g-K_{s}\right)_{0}$ \\
\hline$O_{i}$ & $2.150 \pm 0.199$ & $2.767 \pm 0.270$ & $2.885 \pm 0.267$ \\
\hline$p_{i}$ & $0.194 \pm 0.462$ & $0.097 \pm 0.627$ & $0.130 \pm 0.618$ \\
\hline$r_{i}$ & $0.008 \pm 0.012$ & $-0.021 \pm 0.017$ & $-0.015 \pm 0.016$ \\
\hline$s_{i}$ & $0.531 \pm 0.050$ & $0.556 \pm 0.068$ & $0.590 \pm 0.067$ \\
\hline$R$ & 0.977 & 0.972 & 0.975 \\
\hline$s$ & 0.081 & 0.110 & 0.108 \\
\hline \multicolumn{4}{|c|}{ Equations independent from metallicity, $i=4,5,6$} \\
\hline & $(g-J)_{0}$ & $(g-H)_{0}$ & $\left(g-K_{s}\right)_{0}$ \\
\hline$o_{i}$ & $2.172 \pm 0.196$ & $2.706 \pm 0.267$ & $2.842 \pm 0.262$ \\
\hline$p_{i}$ & $0.160 \pm 0.457$ & $0.185 \pm 0.626$ & $0.192 \pm 0.614$ \\
\hline$s_{i}$ & $0.514 \pm 0.043$ & $0.600 \pm 0.058$ & $0.622 \pm 0.057$ \\
\hline$R$ & 0.976 & 0.972 & 0.975 \\
\hline$s$ & 0.081 & 0.110 & 0.108 \\
\hline
\end{tabular}

\subsection{Residuals}

We compared the observed colors with those evaluated via Eqs. (6)-(29). The residuals are plotted versus observed $(B-$ $V)_{0},(J-H)_{0}$ or $(g-r)_{0}$ colors in Fig. 5. There are some the $(B-V)_{0}$ color. However, they are negligible when compared to the accuracy of the transformations. Actually, the mean of the residuals are smaller than a thousandth and the standard deviations are close to $0.1 \mathrm{mag}$ for all colors (Table 4). The ranges of the residuals for different colors are not the same. The residuals are larger for colors over wider wavelength intervals but they are smaller for metal dependent transformations than the corresponding ones for metal independent transformations. One can confirm this argument by comparing the ranges of the residuals in Fig. 5 and the standard deviations in Table 4. small systematic deviations in the the red and blue ends of

and furthermore into the SDSS system. Thus, this is the complement of the paper of Bilir et al. (2008) where transformations were carried out for dwarfs. We adopted the following steps in order to obtain accurate transformations: (1) the sample was selected by means of the star surface gravities, i.e. $2<\log g \leq 3$, (2) the photometric data was dereddened, (3) stars in the sample were selected according to their photometric data quality, (4) transformations were constructed as two-color dependent equations. Step (3) is especially important for 2MASS data because the inherent errors are larger relative to those in other photometries. Additionally, the transformations are given either as a function of metallicity or independent from metallicity. However, in the case of metallicity dependent equations the procedure is different than the one of Bilir et al.'s (2008). Instead of separating the star sample into different metallicity intervals, we added the metallicity as a term into the transformation equations.

The coefficients of the color terms in the same transformation equation are significant (see Tables 2 and 3) which indicate that the transformations are two-color dependent. On the other hand, the correlation coefficients for the metallicity dependent transformations are larger than the ones of metallicity independent transformations indicating that the transformations actually vary with metallicity. The mean of the residuals, i.e. the color differences between those measured and those calculated, are smaller than $0.001 \mathrm{mag}$ (except for the color $(r-J)$ which is equal to $0.001 \mathrm{mag}$ ). Additionally, the deviations of the measured colors from the calculated ones are absolutely smaller $(s \leq 0.16)$ than the ones cited by Bilir et al. (2008) for dwarfs $(0.2<s \leq 0.3)$.

We applied our transformation equations to the synthetic $B V I$ data of Pickles (1998) which cover stars of a wide spectral type range, and obtained $J-H, H-K_{s}, g-r$ and $r-i$ colors (Table 5) which gave us the chance to compare the two color diagrams in different studies. In all panels of Fig. 3, the grey circles correspond to the positions of our sample stars, whereas the dashed lines in panels (a), (b) and (c) represent the calibrations of Pickles (1998), Covev et al. (2008) and Straizys \& Lazauskaite (2009), respectively. Finally, the continuous lines in panels (b) and (c) correspond to the two-color calibrations evaluated using the converted colors in Table 5. The deviation between the dashed and continuous lines in panel (b) originates from deviations of the positions of our sample stars from the synthetic $(B-V)$ and $(V-I)$ colors in panel (a). But the difference between two lines in panel (c) may be due to the uncertainty of the calibration itself, in addition to the mentioned deviations. However, we can argue that the transformations derived for late type giants in this work could be applied with sufficient accuracy.

\section{Acknowledgments} We present color transformations for late-type giants from We thank the anonymous referee for a thorough report and
2MASS photometric system into the Johnson-Cousins'system useful comments that helped in improving an early version 

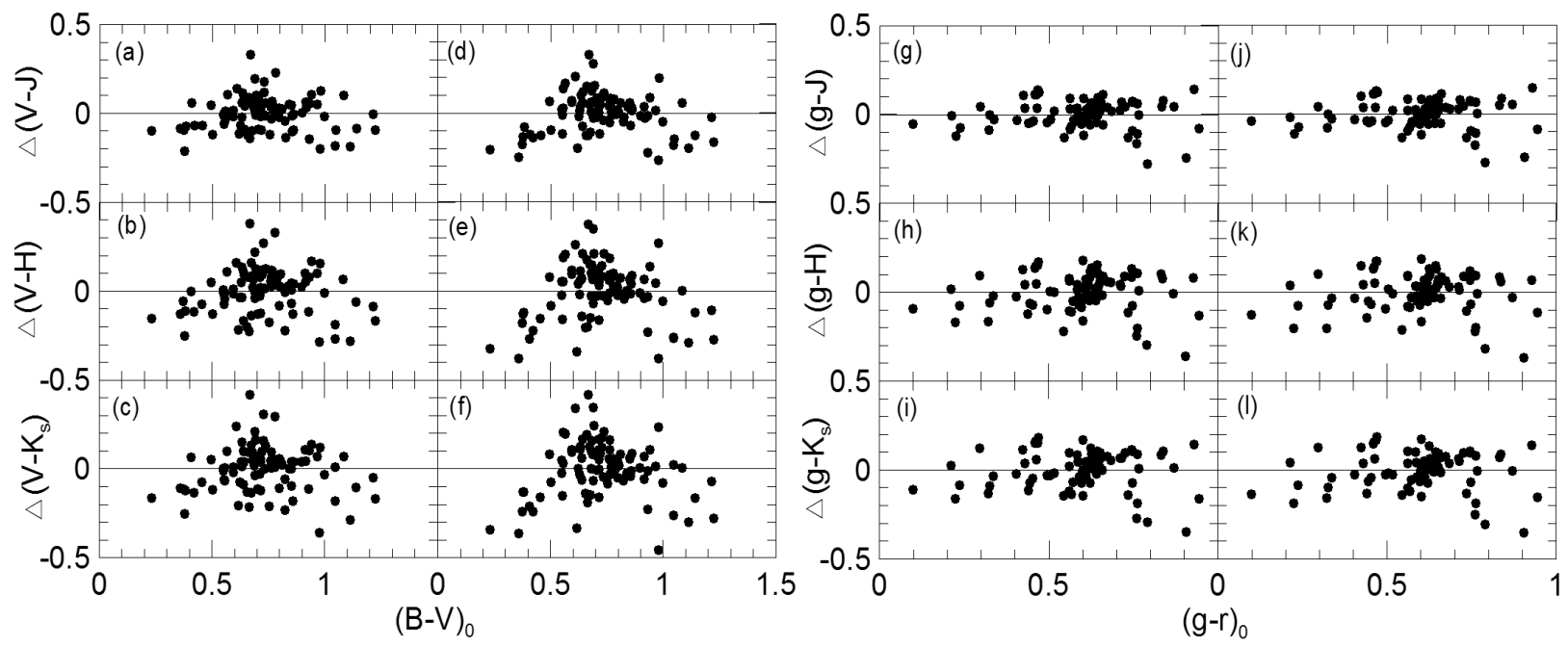

Fig. 5 Color residuals for two sets of transformations. The notation used is $\Delta$ (color)=(evaluated color)-(measured color). The first and third columns correspond to the metal dependent transformations, whereas residuals for metallicity independent transformations are given in the second and fourth columns.

Table 3 Averages and standard deviations $(s)$ for the residuals for different colors in transformation Eqs. (6)-(29). The notation used is $\Delta$ (color) $=$ (measured color) - (evaluated color).

\begin{tabular}{|c|c|c|c|c|c|c|}
\hline \multicolumn{7}{|c|}{ For metallicity dependent transformation equations } \\
\hline & $\Delta(B-J)$ & $\Delta(V-J)$ & $\Delta(I-J)$ & $\Delta(g-J)$ & $\Delta(r-J)$ & $\Delta(i-J)$ \\
\hline Average & 0.0007 & 0.0001 & 0.0006 & 0.0001 & 0.0010 & -0.0004 \\
\hline$s$ & 0.142 & 0.089 & 0.077 & 0.106 & 0.073 & 0.069 \\
\hline & $\Delta(V-J)$ & $\Delta(V-H)$ & $\Delta\left(V-K_{s}\right)$ & $\Delta(g-J)$ & $\Delta(g-H)$ & $\Delta\left(g-K_{s}\right)$ \\
\hline Average & 0.0001 & -0.0004 & 0.0002 & -0.0007 & 0.0001 & 0.0002 \\
\hline$s$ & 0.097 & 0.133 & 0.133 & 0.081 & 0.110 & 0.108 \\
\hline \multicolumn{7}{|c|}{ For transformation equations independent from metallicity } \\
\hline & $\Delta(B-J)$ & $\Delta(V-J)$ & $\Delta(I-J)$ & $\Delta(g-J)$ & $\Delta(r-J)$ & $\Delta(i-J)$ \\
\hline Average & 0.0003 & 0.0001 & 0.0001 & 0.0003 & 0.0001 & 0.0003 \\
\hline \multirow{2}{*}{ 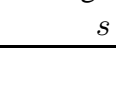 } & 0.167 & 0.092 & 0.080 & 0.124 & 0.083 & 0.075 \\
\hline & $\Delta(V-J)$ & $\Delta(V-H)$ & $\Delta\left(V-K_{s}\right)$ & $\Delta(g-J)$ & $\Delta(g-H)$ & $\Delta\left(g-K_{s}\right)$ \\
\hline Average & -0.0005 & -0.0001 & -0.0001 & 0.0004 & -0.0001 & -0.0005 \\
\hline$s$ & 0.113 & 0.160 & 0.162 & 0.081 & 0.110 & 0.108 \\
\hline
\end{tabular}

Table 4 Synthetic data taken from Pickles'(1998) (columns 1-5) and $J-H, H-K_{s}, g-r$ and $r-i$ colors evaluated by the corresponding transformations derived in this work.

\begin{tabular}{ccccccccc}
\hline Spectral Type & {$[M / H](\mathrm{dex})$} & $T_{\text {eff }}(\mathrm{K})$ & $B-V$ & $V-I_{c}$ & $J-H$ & $H-K_{s}$ & $g-r$ & $r-i$ \\
\hline A0III & 0 & 9572 & 0.037 & 0.008 & -0.003 & 0.026 & 0.027 & -0.050 \\
A3III & 0 & 8974 & 0.130 & 0.076 & 0.040 & 0.043 & 0.102 & -0.019 \\
A5III & 0 & 8453 & 0.175 & 0.164 & 0.068 & 0.040 & 0.133 & -0.006 \\
A7III & 0 & 8054 & 0.210 & 0.240 & 0.091 & 0.035 & 0.156 & 0.003 \\
F0III & 0 & 7586 & 0.271 & 0.317 & 0.123 & 0.040 & 0.202 & 0.022 \\
F2III & 0 & 6839 & 0.417 & 0.445 & 0.194 & 0.062 & 0.317 & 0.069 \\
F5III & 0 & 6531 & 0.425 & 0.507 & 0.204 & 0.052 & 0.318 & 0.069 \\
G0III & -0.22 & 5610 & 0.661 & 0.726 & 0.330 & 0.086 & 0.509 & 0.149 \\
G5III & -0.12 & 5164 & 0.886 & 0.872 & 0.428 & 0.131 & 0.690 & 0.223 \\
G8III & 0.06 & 5012 & 0.946 & 0.927 & 0.449 & 0.139 & 0.733 & 0.240 \\
K0III & -0.08 & 4853 & 0.957 & 0.978 & 0.466 & 0.132 & 0.740 & 0.244 \\
K1III & 0.09 & 4656 & 1.036 & 1.044 & 0.496 & 0.145 & 0.800 & 0.268 \\
K2III & 0.05 & 4457 & 1.105 & 1.132 & 0.535 & 0.150 & 0.853 & 0.290 \\
K3III & -0.02 & 4365 & 1.210 & 1.169 & 0.582 & 0.177 & 0.943 & 0.327 \\
\hline
\end{tabular}


E. Yaz et al.: Transformations between 2MASS, SDSS and BVI photometric systems for late-type giants

of the paper. S. Karaali is grateful to the Beykent University for financial support. This publication makes use of data products from the Two Micron All Sky Survey, which is a joint project of the University of Massachusetts and the Infrared Processing and Analysis Center/California Institute of Technology, funded by the National Aeronautics and Space Administration and the National Science Foundation.

This research has made use of the SIMBAD, NASA's Astrophysics Data System Bibliographic Services and the NASA/IPAC Extragalactic Database (NED) which is operated by the Jet Propulsion Laboratory, California Institute of Technology, under contract with the National Aeronautics and Space Administration.

\section{References}

Abazajian K., Adelman-McCarthy J.K., Ageros M.A., Allam S.S., Anderson K., Anderson S.F., Annis J., Bahcall N.A., Baldry I.K., Bastian S., and 143 coauthors: 2004, AJ 128, 502

Axer M., Fuhrmann K., Gehren T.: 1994, A\&A 291, 895

Bahcall J.N., Soneira R.M.: 1980, ApJS 44, 73

Barbuy B., Erdelyi-Mendes M.: 1989, A\&A 214, 239

Bilir S., Ak S., Karaali S., Cabrera-Lavers A., Chonis T.S., Gaskell C.M.: 2008, MNRAS 384, 1178

Burris D.L., Pilachowski C.A., Armandroff T.E., Sneden C., Cowan J.J., Roe H.: 2000, ApJ 544, 302

Cabrera-Lavers A., González-Fernández C., Garzón F., Hammersley P. L., \& López-Corredoira M.: 2008, A\&A 491,781

Carney B.W., Wright J.S., Sneden C., Laird J.B., Aguilar L.A., Latham D.W.: 1997, AJ 114, 363

Cavallo R.M., Pilachowski C.A., Rebolo R.: 1997, PASP 109,226

Cayrel de Strobel G., Soubiran C., Ralite N.: 2001, A\&A 373, 159

Chonis T.S., Gaskell C.M.: 2008, AJ 135, 264

Clementini G., Gratton R.G., Carretta E., Sneden C.: 1999, MNRAS 302, 22

Covey K.R., Hawley S.L., Bochanski J.J., West A.A., Reid I.N., Golimowski D.A., Davenport J.R.A., Henry T., Uomoto A., Holtzman J.A.: 2008, AJ 136, 1778

Cutri R. M., Skrutskie M.F., Van Dyk S., Beichman C.A., Carpenter J.M., Chester T., Cambresy L., Evans T., Fowler J., Gizis J., Howard E., Huchra J., Jarrett T., Kopan E.L., Kirkpatrick J.D., Light R.M, Marsh K.A., McCallon H., Schneider S., Stiening R., Sykes M., Weinberg M., Wheaton W.A., Wheelock S., Zacarias N.: 2003, 2MASS All-Sky Catalog of Point Sources, CDS/ADC Electronic Catalogues 2246

Davenport J.R.A., Bochanski J.J., Covey K.R., Hawley S.L.,West A.A., Schneider D.P.: 2007, AJ 134, 2430

Dominy J.F.: 1984, ApJS 55, 27

Drake J.J., Lambert D.L.: 1994, ApJ 435, 797

ESA: 1997, The Hipparcos and Tycho Catalogues ESA SP1200. ESA, Noordwijk
Fan X.: 1999, AJ 117, 2528

Fiorucci M., Munari U.: 2003, A\&A 401, 781

Fukugita M., Ichikawa T., Gunn J.E., Doi M., Shimasaku K., Schneider D.P.: 1996, AJ 111, 1748

Fulbright J.P., Kraft R.P.: 1999, AJ 118, 527

Fulbright J.P.: 2000, AJ 120, 1841

Gilroy K.K., Sneden C., Pilachowski C.A., Cowan J.J.: 1988, ApJ 327, 298

Gratton R.G.: 1983, A\&A 123, 289

Gratton R.G., Ortolani S.: 1984, A\&A 137, 6

Gratton R.G.: 1989, A\&A 208, 171

Gratton R.G., Sneden C.: 1991, A\&A 241, 501

Gratton R.G., Sneden C., Carretta E., Bragaglia A.: 2000, A\&A 354, 169

Gunn J.E., Carr M., Rockosi C., Sekiguchi M., Berry K., Elms B., de Haas E., Ivezic Z., Knapp G., Lupton R., and 30 coauthors: 1998, AJ 116, 3040

Hogg D.W., Finkbeiner D.P., Schlegel D.J., Gunn J.E.: 2001, AJ 122, 2129

Jordi K., Grebel E.K., Ammon K.: 2006, A\&A 460, 339

Karaali S., Bilir S., Tunçel S.: 2005, PASA 22, 24

Kovacs N.: 1983, A\&A 120, 21

Kraft R.P., Sneden C., Langer G.E., Prosser C.F.: 1992, AJ 104,645

Krishnaswamy K., Sneden C.: 1985, PASP 97, 407

Landolt A.U.: 1992, AJ 104, 340

Leep E.M., Wallerstein G.: 1981, MNRAS 196, 543

López-Corredoira M., Cabrera-Lavers A., Garzón F., \& Hammersley P. L.: 2002, A\&A 394, 883

Lucas P.W., Hoare M.G., Longmore A., Schrder A.C., Davis C.J., Adamson A., Bandyopadhyay R.M., de Grijs R., Smith M., Gosling A., and 21 coauthors: 2008, MNRAS 391, 136

Luck R.E., Bond H.E.: 1985, ApJ 292, 559

Luck R.E., Bond H.E.: 1991, ApJS 77, 515

Luck R.E., Wepfer G.G.: 1995, AJ 110, 2425

Luck R.E., Challener S.L.: 1995, AJ 110, 2968

Lupton R.H., Gunn J.E., Ivezic Z., Knapp G.R., Kent S., Yasuda N.: 2001, in ASP Conf. Ser.: Astronomical Data Analysis Software and Systems X, ed. F. R. Harden Jr., F.A. Primini and H.E. Payne, 238, 269

Marshall D.J., Robin A.C., Reylé C., Schultheis M., Picaud S.: 2006, A\&A 453, 635

Mishenina T.V., Klochkova V.G., Panchuk V.E.: 1995, A\&AS 109, 471

Ofek E.O.: 2008, PASP 120, 1128

Peterson R.C.: 1981, ApJS 45, 421

Pickles A.J.: 1998, PASP 110, 863

Pilachowski C.A., Sneden C., Kraft, Robert P.: 1996, AJ 111,1689

Rodgers C.T., Canterna R., Smith J.A., Pierce M.J., Tucker D.L.: 2006, AJ 132, 989

Ryan S.G., Norris J.E., Bessell M.S.: 1991, AJ 102, 303

Ryan S.G., Lambert D.L.: 1995, AJ 109, 2068

Ryan S.G., Deliyannis C.P.: 1998, ApJ 500, 398 
Schlegel D.J., Finkbeiner D.P., Davis M.: 1998, ApJ 500, 525

Shetrone M.D.: 1996, AJ 112, 1517

Skrutskie M.F., Cutri R.M., Stiening R., Weinberg M.D., Schneider S., Carpenter J.M., Beichman C., Capps R., Chester T., Elias J., and 21 coauthors et al.: 2006, AJ 131, 1163

Smith V.V., Lambert D.L.: 1986, ApJ 303, 226

Smith J.A., Tucker D.L., Kent S., Richmond M.W., Fukugita M., Ichikawa T., Ichikawa S., Jorgensen A.M., Uomoto A., Gunn J.E.: 2002, AJ 123, 2121

Smith J.A., Tucker D.L., Allam S.S., Ivezic Z., Yanny B., Gunn J.E., Knapp G.R., Eisenstein D., Finkbeiner D., Fukugita M.: 2007, The Future of Photometric, Spectrophotometric and Polarimetric Standardization, ASP Conference Series, Vol. 364, Edited by C. Sterken, San Francisco: Astronomical Society of the Pacific, p. 91

Spite M., Spite F.: 1980, A\&A 89, 118

Spite M., Molaro P., Francois P., Spite F.: 1993, A\&A 271L, 1

Spite, M., Pasquini, L., Spite, F.: 1994, A\&A 290, 217

Straizys V., Lazauskaite R.: 2009, BaltA 18, 19

Tomkin J., Lemke M., Lambert D.L., Sneden C.: 1992, AJ 104,1568

Tomkin J., Lambert D.L.: 1999, ApJ 523, 234

Tucker D.L., Kent, S., Richmond, M.W., Annis, J., Smith, J.A., Allam, S.S., Rodgers, C.T., Stute, J.L., AdelmanMcCarthy, J.K., Brinkmann, J., and 24 coauthors: 2006, AN 327, 821

van Leeuwen F.: 2007, A\&A 474, 653

Walkowicz L.M., Hawley S.L., West A.A.: 2004, PASP 116, 1105

West A.A., Walkowicz L.M., Hawley S.L.: 2005, PASP 117, 706

Yadav R.K.S., Sagar R.: 2004, MNRAS 349, 1481

York D.G., Adelman J., Anderson J.E.Jr., Anderson S.F., Annis J., Bahcall N.A., Bakken J.A., Barkhouser R., Bastian S., Berman E., and 134 coauthors: 2000, AJ 120, 1579 